\title{
A Distributed Environment with Rough Set Theory Based Image Processing Approach for Analysis of Facial Disorders for Better Cosmetic Product Recommendation
}

\author{
Pakalapati Manikya Prasuna $^{*}$, Yellasiri Ramadevi², Arramraj Vinaya Babu ${ }^{3}$ \\ ${ }^{1}$ Department of Computer Science \& Engineering, Jawaharlal Nehru Technological University, Hyderabad 500085, Telangana, \\ India \\ ${ }^{2}$ Department of Computer Science \& Engineering, Chaitanya Bharathi Institute of Technology, Hyderabad 500075, Telangana, \\ India \\ ${ }^{3}$ Department of Computer Science \& Engineering, Stanley College of Engineering and Technology for Women, Hyderabad \\ 500001, Telangana, India
}

Corresponding Author Email: prasunamanikya@gmail.com

https://doi.org/10.18280/ijsse.100608

Received: 5 October 2020

Accepted: 10 December 2020

\section{Keywords:}

image processing, rough set theory, facial disorders, product recommendation, feature selection

\begin{abstract}
Producers and Consumers are getting progressively open to the use of cosmetic beauty care products. This can be found in them utilizing an assortment of regular Cosmetic resources and materials. This reality is additionally upheld by the pattern of natural and health awareness. This experience can be found inside both the Producers and Consumers behavior. Fast development of beauty care products industry, the improvement of new advances and the style for wonderful and youthful skin caused that fashion became basics for present day society. As the growth of cosmetic industry increases, the number of cases related to side effects of the products are also getting increased. According to Central Drugs Standard Control Organization, nearly $8 \%$ of the customers are facing side effects because of unsuitable products. There is no proper model used by the cosmetic product producers to suggest suitable cosmetic product to the customers. To reach the requirements of the various customers, the cosmetic industry needs to manage enormous features of skin, extract from the face images of the customers, out of which not many are required to recognize the skin issues and fundamental item stimulation. The proposed work mainly concentrates on designing a model for better cosmetic product suggestions to the customers to reduce the side effects and to have a healthy and shining skin. Advanced image handling and examination of clinical face images can successfully strengthen medical diagnosis with important plans including automatic prediction and analysis, image segmentation and estimation of obvious features in images. Rough set theory is one of numerous techniques that can be utilized to break down and analyze images, less regular than progressively customary strategies for probability, statistics and set theory. In this manuscript a distributed environment with rough set theory based image processing method is used for analysis of facial disorders for better cosmetic product recommendation that cannot cause any harm or disorder to the customer with use of cosmetic products that improves the safety of the customers. The proposed model performs product recommendations based on local and global features based on the customer locality. The proposed method is compared with the traditional methods and the results show that the proposed method exhibits better accuracy in prediction of skin disorders in cosmetic industry.
\end{abstract}

\section{INTRODUCTION}

The usage of Cosmetic items and the markets manufacturing such products are increasing in recent decades as customers are taking very much care about their beauty in present lifestyle. Customers purchase beauty care products for purging, improving or changing the appearance, skin, hair and nails especially face tone improving products. Cosmetic items incorporate excellence arrangements, for example, make-up and face cream products that helps, for example, cleanser and antiperspirant [1]. At the point when herbs are utilized for their fragrant and cosmetic usage in makeup, they are known as home developed or green entity consideration items.

The historical backdrop of cosmetic products has experienced radical changes during the previous decades. The worldwide cosmetic market was around 590 billion USD in 2019 and it is assessed to arrive at 750 billion USD by end of 2020 , developing at a rate of 20 percent [2]. The Beauty advertisements has developed by 15 percent a year on a normal with a yearly development rate running from 8 percent to 10 percent. During the 20th century, India built up a fabrication procedure to replace the scent, cosmetic products made by regular and with chemical techniques [3].

The Indian cosmetic industry experienced quick development rate since the monetary Liberalization in 1991. All through the main decade of 21 st century, the offer of Cosmetic products became consistently arriving at the degree of 9 percent Compound Annual Growth Rate (CAGR) in the 
primary half and 10 Percent (CAGR) in the later half. The excellence business was influenced in 2010 and recovered in 2018 because of the improvement of worldwide economy [4]. The Cosmetic business is presently a multibillion-dollar industry. India is the second biggest exporter of moderate beautifiers to the world market after China [5]. The Indian cosmetic industry has seen a quick development throughout the most recent few decades when the Indian contenders began fabricating items so as to meet the expanded needs of both the Indian and the global markets [6]. Huge numbers of the world's mainstream Cosmetic brands entered the Indian market in 1990s so as to satisfy the solid need for the corrective items in India [7]. The facial disorders caused due to the usage of cosmetic products that causes reactions are depicted in Figure 1.

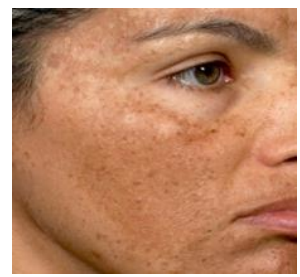

Figure 1. Cosmetic product reaction

As the utilization of cosmetic products are increasing day by day, manufacture companies have to take much care in producing the cosmetic products that best suits the customers and satisfy their requirements. Because of the usage of various chemicals and herbs, a lot of care need to be taken as the product should not cause any harm or any reaction to the users. In India, there is 4 percent of cases that represents the reactions caused to customers especially face disorders or facial reactions [8]. As the research in the field of cosmetic industry is a growing field, researchers have to provide platforms for suggesting cosmetic products to the customers only after analyzing the skin behavior [9]. In India, many research organizations are performing several experiments on this area and still many customers are facing facial or skin disorders that is a worrying issue [10]. In the proposed work, a distributed environment with rough set theory based image processing approach for analysis of facial disorders for better cosmetic product recommendation is introduced that helps the customers to provide their face as input and check which cosmetic product best suits their skin that does not cause any harm or reaction to them.

Customer purchasing behavior is the total of a buyer's perspectives, inclinations, aims, and choices with respect to the product manufacturer history in the commercial center when buying an item [11]. The investigation of customer conduct draws upon sociology orders of human studies, brain research, social science, and financial matters. Taste and tradition of the general public have changed because of expanding familiarity with the western world and excellence patterns and more productive employments [12]. Accordingly women from such social layers are currently increasingly aware of their appearance and is happy to spend additional cash on it further. The adjustment in Cosmetic and healthy skin item utilization is seen today because of ascend women population, particularly from the employees having increasingly flexible cashflow. This really has improved development in certain item classifications in the market that was seen before [13]. The beauty care products and sun care items that have demonstrated development paces of $51 \%$ and
$16 \%$ individually in the recent years [14].

The scope of Cosmetic items in India has broadened hugely in recent years [15]. Cosmetic item makers in India have for the most part been obliging the extraordinary interest for products that fall into the low-or medium priced classes as the best interest in India consistently spin around financially estimated items [16]. The segment has chiefly been driven by improved buying interest and rising style based on the Indian populace. Besides, to attract customers the marketing agencies are promptly spending on the special exercises [17]. The product recommendation method strictly identifies the customer's skin condition by applying image processing technique and extracting features from the skin and comparing the features with the existing data of cosmetic reactions data and suggesting customers the best product that will not cause any harm or reactions to users [18]. Taking such care on the customers will definitely improve business levels and also the customers show their interest in buying cosmetic products without any uncertainty or fear thus improves the customer needs [19].

\section{LITERATURE SURVEY}

Sigma Research Indonesia conducts research on 1,200 ladies matured 15-55 years. From these investigations they identified that there are a few elements for ladies in buying corrective items. The greatest factor is the rate coordinates facial skin, trailed by enduring items, lightweight chemicals fit, with rate cost by considering brand also. Patty et al. [1] introduced a proposal framework is a model utilization of the aftereffects of perceptions on the conditions and reactions on the client. In this way, a framework requires a model of exact suggestions that were suggested as per the desires of clients, just as empowering clients to take the correct choice in figuring out which item will be utilized.

Ekstrand et al. [2] discovered huge distinction between the use and disposition of the groups of customers. This investigation came about into the development of three clusters viz. medium self-coordinated, substantial othercoordinated changing attributes. Furthermore, brand inclination for customer products was found to change by group and brand inclination for face wash, shaving cream and face cream as multiple clusters. Shockingly, substantial other ingredients were accounted for to be the least spenders while incidental users were the most impacted.

Sharma and Gera [3] asserts the more extensive phases of a purchaser's dynamic procedure that incorporates issue ID, data search, evaluation of choices, outlet determination and buying selected products and post-buy activity (fulfillment). With respect to facial healthy skin, the variables that impact the purchaser would include: convictions in product property, natural concern, segment and individual trademark which initiate the shopper to buy the facial skin health management product.

Yasir et al. [4] directed an investigation and arrived at a resolution that ladies who go through uncertainty and are on edge and need certainty about them. Cosmetic items contain different unsafe or poisonous synthetic substances fit for making hurtful impacts on the skin. Makers of Cosmetic items don't just utilize manufactured fixings yet in addition common items, for example, Shea margarine, Rose concentrate, and natural sweetener which are manageable, modest and less harmful to the customer. Skincare items, for example, 
fragrances, make up, nail cleaners and so on, stay on the skin for a more drawn out timeframe and can cause unsafe impacts like hypersensitive responses. Moisturizers increment the hygroscopic properties of the skin especially when utilized in high fixation. It can cause inconvenience and peeling on the skin.

Schneider et al. [8] recommend that a multi-dimensional methodology of understanding excellence is increasingly suitable, instead of the single dimensional methodology of alluring-ugly utilized by analysts to assess people groups view of magnificence for suggestion of products. Portugal et al. [9] talks about how advertising utilization of appealing individuals in promoting for an exchange with customers has strongly affected purchasers impression of their own facial excellence.

Robertson et al. [11] proposed a framework model with utilization of the aftereffects of perceptions on the conditions of the client. In this way, a framework requires a model of exact suggestions that were suggested as per the desires of clients, just as empowering clients to take the correct choice in figuring out which items will be best suitable.

Rinaldi [12] made a suggestion to buy the beauty care products. In this investigation utilizing Content Based Filtering is suggested for product selection. The motivation behind this investigation is to build up an arrangement of Cosmetic proposals for customers who will purchase facial products that suit your necessities as indicated by skin shading and skin type. Content Based Filtering is done dependent on regular methodologies by analyzing skin conditions. At that point the similitude of the product profiles and customer profiles utilizing the Cosine Similarity is done and a product is recommended.

According to Central Drugs Standard Control Organization (CDSCO), the cosmetic products are increasing from year to year by $20 \%$ and customer satisfaction rate is decreasing every year by $15 \%$. Nearly $8 \%$ of the users are facing side effects because of unsuitable products and $3 \%$ of them have serious skin problems. Based on the literature survey done, there is no proper model for suggesting customers with suitable products. The proposed model concentrates on designing a distributed model for customers of different regions to suggest a suitable cosmetic product by analyzing the skin conditions. The proposed model concentrates on the safety of the customers who are interested in using cosmetic products.

\section{PROPOSED METHOD}

The proposed method is designed using rough set theory based image processing approach for analysis of facial disorders for better cosmetic product recommendation. The proposed method initially converts the face images into a set of basis functions which essentially are the principal components of the face images that seeks directions in which it is more efficient to represent the data into numeric format for better analysis. This is mainly useful for decreasing the computational effort. Image processing method is primarily used here to convert image pixels into binary values. Converting the pixels into binary values are helpful for quick analyzing and for accurate training. This process of converting image pixels to binary values improves the accuracy levels. The proposed method reduces the number of features to a more manageable number because face is represented by a large number of pixel values. The features extracted from face images for the identification of a normal pixel and effected pixel are pixel contrast, correlation, entropy, uniformity, pixel energy, face shape, eyes position, nose position, pixel intensity. The proposed model performs training of features which contain normal face images and skin images having side effects. Based on the trained features, if new set of features are applied then side effects can be analyzed. The products are initially used by the customers and their facial features are extracted for training the model.

Each of the new dimensions is a linear combination of pixel values, which form a face template. The linear combinations obtained are an order set of binary comparisons of pixel intensities between the center pixel and its eight surrounding pixels. The pixel extraction process from the image given as input is done as

$$
\begin{aligned}
\operatorname{IDS}(\mathrm{I}(\mathrm{x}, \mathrm{y}))=\sum_{x=1}^{N} \operatorname{mean}(\mathrm{x})+\sum_{y=1}^{N} \operatorname{mean}(\mathrm{y}) \\
+\sum_{n=0}^{M} s\left(i_{x}-i_{y}\right) 2^{N}
\end{aligned}
$$

where, $i_{x}$ and $i_{y}$ corresponds to the value of the center pixel $\left(x_{n}, y_{n}\right), \mathrm{S}\left(i_{x}\right)$ and $\mathrm{S}\left(i_{y}\right)$ represents to the value of eight surrounding pixels, function $\mathrm{f}(\mathrm{x})$ represents the pixel values as 1 if required and 0 if not required that is defined as:

$$
f(x)=\left\{\begin{array}{cc}
1 & \text { if } x>=0 \\
0 & \text { if } x<0
\end{array}\right.
$$

By looking at the extracted rationality vectors of images, a proximity measure is proposed that predicts the relevant and irrelevant pixels in an image. The comparability measure between two image pixels $\mathrm{x}$ and $\mathrm{y}$ is considered with the parameters:

$$
I(\operatorname{diff}(x, y))=\sum_{i=1}^{N}\left|\alpha_{j}-\alpha_{j}^{\prime}\right|(\mathrm{X})+\left|\beta_{j}-\beta_{j}^{\prime}\right|(\mathrm{Y})
$$

where, $\alpha$ and $\beta$ are the color levels of the image that represents dark and light colored pixels. On the basis of color histogram, some statistics to reflect the characteristics of the image pixels are calculated. Mean reflects the average gray values of an image that is calculated as:

$$
M(I(x, y))=\sum_{i=0}^{N} x H(y) * i+f(x)
$$

$\mathrm{H}$ represents the histogram value. Variance reflects the gray value pixels of an image on the numerical discrete distribution, variance is a measure of the width of the histogram, the difference between the average and gray levels of pixels that are extracted.

$$
\sigma^{2}=\sum_{i=0}^{L-1}(i-\mu)^{2} H(i)
$$

Here $\mu$ represents the gray level value of the pixel. Fourier transform model is used to transform the image convolution operation for getting the frequency domain characteristics of the image. For the $\mathrm{P} \times \mathrm{Q}$ original image $\mathrm{I}(\mathrm{x}, \mathrm{y})$, its 
corresponding Fourier spectrum can be expressed as $\mathrm{f}(\mathrm{x}, \mathrm{y})$ and the numerical representation of the image is represented as:

$$
\mathrm{I}(\mathrm{PXQ})=\left[\begin{array}{ccccc}
-\frac{d_{x}+d_{y}}{\alpha n_{x}} & -\frac{n_{x}^{2}+n_{y}^{2}}{\alpha n_{x}} & 0 & 0 & 1 \\
-\frac{d_{x} h_{(\mathrm{x})}+d_{y} h_{(y)}}{I_{\beta} n_{x}} & -\frac{d_{x}+d_{y}}{I_{\beta} n_{y}} & 0 & 0 & 0 \\
-1 & -H(x) & 0 & n_{x} & 0 \\
0 & -H(y) & 0 & 0 & n_{y} \\
0 & 0 & 0 & 0 & 1
\end{array}\right]
$$

Rough Set Theory (RST) which created in mid-1980's, which is having its noteworthiness to data acquirement and gathering through AI techniques, Feature extraction systems etc. One of the most important use of RST is that it needn't disorder data with any kind of preliminary information about the model data assortment for instance probability allocation, estimated probability task, etc. Rough Set Theory is one of the data mining methodologies which reduce the features from huge amounts of data that when applied on cosmetic data can extract only relevant features to suggest the best product to the customers for avoiding reactions to face when applying cosmetic products.

The face disorders of several customers are trained using the representations.

$$
I(X, Y)=\sum_{i=0}^{N-1} H(i)-\text { similarity }\left(i_{x}-i_{y}\right)+K
$$

Every Image 'I' from the dataset is considered I $\varepsilon$ Image Set (IS), $\mathrm{H}$ is the histogram range and $\mathrm{K}$ is the Threshold brightness value to be considered for extraction.

The pixels from the image are extracted and forms a cluster $\mathrm{C}$ based on relevant and irrelevant data.

Consider $\mathrm{C} 1, \mathrm{C} 2, \ldots, \mathrm{C}(\mathrm{N})$ be the $\mathrm{N}$ clusters formed with $\left|C_{i}\right|$ $\leq\left|C_{j}\right|$ and $\mathrm{W}$ is initialized as 1 for starting pixel. For each pixel extracted from the image, weights are assigned to every pixel for establishing similar cluster set. Weights W are calculated as:

$$
\mathrm{W}(\mathrm{I}(\mathrm{x}, \mathrm{y}))=\frac{1}{\sum_{\mathrm{i}}^{\mathrm{N}} \mathrm{W}_{\mathrm{i}}} \sum_{\mathrm{i}}^{\mathrm{N}}\left|x_{i}-y_{j}\right|^{2}+\sum_{j=1}^{N} \sum_{i=1}^{\mathrm{M}}\left|x_{i}^{(j)}-y_{j}^{(i)}\right|^{2}
$$

For rotating the images for maintaining same angle $\theta$ in the range $(-\lambda$,$) of the inverse tangent function of the slope of the$ line connecting the two points for fixing the pixels in a particularangle. The pixel rotation is done as:

$$
\theta=\arctan \left(\frac{y_{2}-y_{1}}{x_{2}-x_{1}}\right)
$$

The facial image considered undergo segmentation process to extract pixels quickly by considering parts of image that is useful for extracting face disorder pixels only. The process is done as:

$$
I_{\sigma}\left(I\left[i_{x}, j_{y}\right]\right)=\sqrt{\left.\sum_{i}^{\mathrm{N}}\left(W-I_{m}(I(x, y)]\right)\right)^{2} \times K}
$$

where, $\mathrm{W}$ is the weight and $\mathrm{K}$ is the threshold value. A model for assigning the weights for the image pixels are used which assigns the weights for the pixels as:

$$
W_{x, y}=\exp \left(\frac{-\left(I_{i}(x)-\mathrm{I}_{n}(y)\right)^{2}}{\left(\frac{\beta^{2}}{2}\right) * \theta}\right)
$$

From the facial image given as input, features are extracted only that forms facial template from a relevant sub image is that is calculated as:

$$
F S(I(x, y))_{\mathrm{N}}=\sum_{W(I) * \mathrm{I}(\mathrm{x}, \mathrm{y})}^{N}\left\{\frac{|| x_{j}-V_{i} \|}{\| x_{j}-V_{K}||}\right\}^{-\frac{2}{m}-1}+
$$

$\mathrm{X}$ and $\mathrm{y}$ are the pixel coordinates, $\mathrm{m}$ is the sub image position, $w$ is the weight of the pixel in the facial template.

After extracting the facial template features, the classifier is trained with the existing facial disorders for better product suggestion for the customers that may not cause any harm or disorder to them. The training process is depicted below.

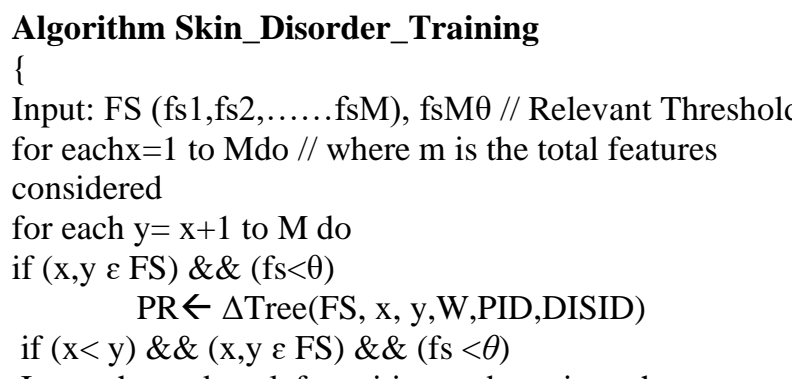

Insert the node at left position and continue the process of inserting

if $(\mathrm{x}>\mathrm{y}) \& \&(\mathrm{x}, \mathrm{y} \varepsilon \mathrm{FS}) \& \&(\mathrm{fs}<\theta)$

Insert the node at right position and continue the process of inserting

if $\mathrm{FS}<\theta$

if (f i $\varepsilon$ FS(M)) \&\& (f i $<\theta) \& \&(x<y)$

search for product id and disorder id in left positions

else

$\mathrm{PR} \leftarrow \Delta$ Tree $(\mathrm{FS}, \mathrm{x}, \mathrm{y}, \mathrm{W}, \mathrm{PID}, \mathrm{DISID})$

end if

foreach $p \in \mathrm{FS}(\mathrm{p})$ do

$\mathrm{W}(\mathrm{P})=\operatorname{Count}($ left $($ DISID $)) / \operatorname{Count}($ left(PID)

Display W(P), FS

\}

where, FS is the feature set, $\mathrm{x}, \mathrm{y}$ are pixel co ordinates, $\mathrm{W}$ is the weight of the feature set and PID is the product id, DISID is the disorder ID. Based on the disorder ID, the products are categorized as recommended or not recommended. When a customer needs any recommendations for a cosmetic product, they need to provide their facial image as input. The classifier then analyze the facial pixels and based on the trained experience, best product that does not cause any disorder will be suggested to the customer who belongs to any nation or locality.

\subsection{Global and local rule setting by central authority for distributed model}

As the proposed application is recommended for better cosmetic product suggestion, there is a chance for this 
application to be useful for all regions and nations of people which is very much helpful to avoid skin disorders and to suggest a suitable product based on analyzing their skin condition. The skin conditions, features of people are different but, the proposed method has to suggest the products to local as well as global level customers. To perform this task, local and global rule setting for feature extraction and product recommendation becomes a challenging task. The proposed method imposes some local and global rules on the images provided for customers for getting better product suggestions. There is less complexity in setting local rules for the skin conditions of the users where as global rule setting is a complex task. The local rule setting on the images are performed and the local rules are analyzed by the central authority. Different cosmetic products are available at different regions and each system applies its rules and then the local rules are applied.

To perform local and global distribution, the proposed model needs to be trained with the features of customers of different regions. The proposed model initially provides an option for the user to select for local or global product suggestion. The proposed model is trained with the local features like customer facial shape, color, contrast, pixel intensity, brightness level, facial edge pixels. The proposed model is also trained with customers of different regions to provide a distributed approach also. The main idea of the proposed model is to recommend cosmetic products to customers at different regions in the world. Facial features of customers will change from region to region. The proposed model should be trained with different datasets of different regions considering normal skin features and infected skin image features. This allows the model to suggest a better cosmetic product for the customers. The customer can select either local or global model based on the locality of the user.

The image given as input undergo the process of preprocessing and feature selection and then, the facial template is fixed by setting the property moving as:

$$
F T \in_{m v}=\sum_{i=\left\{\frac{F S}{2}\right\}+1}^{N}\left(\begin{array}{l}
\theta \\
i
\end{array}\right) \mathrm{I}(\mathrm{x}, \mathrm{y}) \in^{i}(1-E)^{N-i}
$$

where, FS is th feature set, $\theta$ is the angle of the input image, $x$ and $y$ are pixel coordinates, $€$ is the value fixed for balancing the pixels for every customer that depends on the facial template pixels intensity. The local rules are imposed for balacing the image angle, intensity, sub image position and grey levels. The Grey Level Parameter Balancing (GRPB) is done as:

$$
\begin{aligned}
& \operatorname{GRPB}(y)=\sqrt{\frac{\sum_{i=1}^{N} \cdot\left(I_{\text {proj }}-x_{\text {orig }}\right)^{\theta}}{\operatorname{Total}(F S)}} \\
& \operatorname{GRPB}(x)=\sqrt{\frac{\sum_{i=1}^{N} \cdot\left(I_{\text {proj }}-y_{\text {orig }}\right)^{\theta}}{\operatorname{Total}(F S)}}
\end{aligned}
$$

The intensity values for the images are balanced as:

$$
\begin{gathered}
\mathrm{I}(\mathrm{P}(\mathrm{I}))=\operatorname{argmin}_{W}\left(\sum_{i}^{N} \theta \mathrm{FS}(\mathrm{I}(\mathrm{x}, \mathrm{y}))-\left(I_{j}(x)-\right.\right. \\
\left.\mathrm{I}_{i}(y)\right)^{\theta}
\end{gathered}
$$

where, $\mathrm{FS}\left(\mathrm{I}(\mathrm{x}, \mathrm{y})_{i j}\right.$ is the correlation between the $i^{\text {th }}$ and the $j^{\text {th }}$ individual pixel at any angle $\theta$.

As different users from different locations needs cosmetic product recommendations, all this cosmetic product suggestion features selection data has to be monitored and analyzed. To perform data analysis of local product suggestions, a central authority need to be established which has to analyze all the collected local product suggestion features and need to be applicable to global rules so that customers can get better product recommendation. The process of gathering local data by the central authority and setting of global rules is depicted in Figure 2.

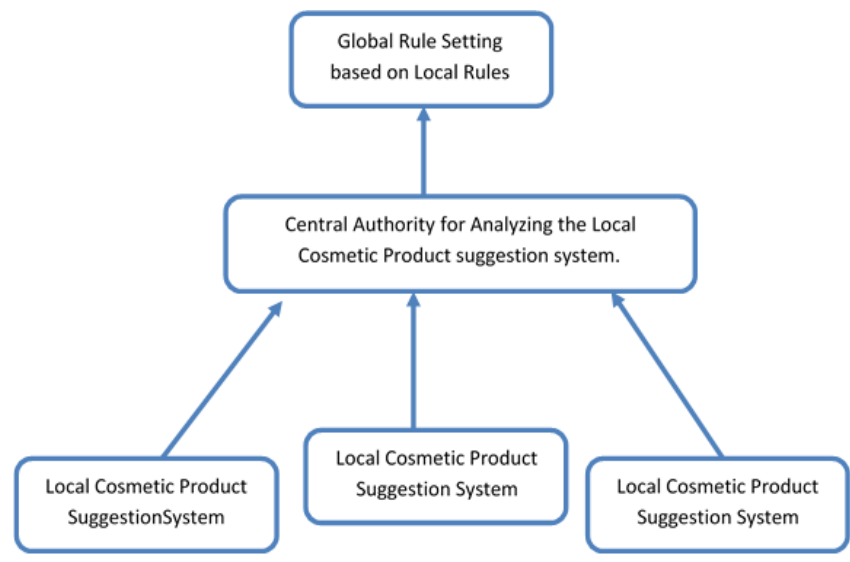

Figure 2. Framework for analyzing local rules and setting global rules

The process of analyzing the local rules by the central administrator is clearly illustrated. Initially the features selected from every product recommender system has to be transferred to the central administrator. The central administrator gathers relavant features trained data and arranges accoringly as data clusters. The process of clustering is done as:

$$
C S^{L D}=\sum_{i}^{N} F s_{i+j}(R(i))+\log \{P(I D)(N)\}^{*} W(P)
$$

Here CS is the cluster set generated based on local data LD, Fs is the features used in the local data, $\mathrm{P}(\mathrm{ID})$ is the product ID and $\mathrm{W}$ is the weights assigned to products locally. All local features are grouped based on the selected feature weights and local rules are grouped together to improve the product suggestions by applying global rules. The weights are newly correlated in the global data cluster set as:

$$
C S^{G D}=\sum_{i}^{N} \sum_{j}^{M}(W-m e a n(P(i, i+1)))^{N}+(\mathrm{j}(\mathrm{x})+\mathrm{i}(\mathrm{y})+\mathrm{L}
$$

Here CS is the cluster set generated based on global data GD. $\mathrm{N}$ is the maximum local rules and $\mathrm{M}$ is the global rule count. $\mathrm{W}$ is the weight of the feature and $\mathrm{P}$ is the Product ID. $\mathrm{I}$ and $\mathrm{j}$ are the relevant features in the subset. $\mathrm{L}$ is the constant for balancing the global rules. The images given as input when compared to local regions is easy to process when compared to global regions. The central authority will finally group all the local rules to make the rules available globally, suchthat every user at any location can use the product recommender system. The central authority finally groups all the local rules as: 


$$
C S(G R)=\sum_{i} \sum_{j} \frac{W(P(I D))+\text { mean }(\mathrm{i}(\mathrm{x})-\mathrm{i}(\mathrm{y})}{1+W(i-j)}
$$

The global regions are then processed after completing and applying local rules as global rule setting concentrates on features extracted and facial template fixing and arranging it same for all kinds of users. The global rule setting model extracts some more features from the images such that every customer at any region can get best product recommendations.

The optimum weight of an image $O W^{\mathrm{I}}$ is calculated for extracting more relevant features and excluding irrelevant features from the given input image is done as:

$$
O W_{N}^{I}=\frac{\sum_{i}^{N} \sum_{j}^{\mathrm{M}}\left|x_{i}^{(j)}-y_{j}^{(i)}\right|^{2}}{\sum_{\substack{i \\+\left|\beta_{j}-\beta_{j}^{\prime}\right|}}^{N} \sum_{j=1}^{M} W_{k j}^{\theta}\left|\alpha_{j}-\alpha_{j}^{\prime}\right|}
$$

The sigmoid function for balancing the facial template structure is balanced as:

$$
\operatorname{Sig}(I(x, y))=\frac{1}{1+e^{-10 \sum_{i=0}^{L-1}(i-\mu)^{2} H(i)\left(H_{i}^{n}-0.5\right)^{\theta}}}
$$

where, $H_{i}^{n}, \mathrm{H}(i)$ are calculated for identification of similarity levels of the global and local images. The image pixels are balanced for all kinds of images for allowing customers at different regions to support distributed environment.

\section{RESULTS}

The Proposed method is implemented in ANACONDA SPYDER platform that takes the input from the customer and analyze the image by performing feature extraction and identifying disorders on the face and training the model with the disorder levels and suggesting the best product to the customer that does not cause any reaction based on the comparisons done with the trained data. The proposed method is compared with the traditional methods and the results show that the proposed method product suggestion is accurate that does not cause any disorders to the customer. The proposed is compared with traditional methods in terms of customer causing disorder rate by using the products, time for analyzing the disorders on the skin. The facial disorder datasets are considered from the link https://archive.ics.uci.edu/ml/datasets/dermatology. The proposed method identifies the number of cases caused by the usage of cosmetic products and reduces the cases by suggesting suitable products to the customers.

The disorders caused from the cosmetic products is considered and analyzed from the customers and depicted in Table 1.

The proposed method is used to train the model based on the available trained data of facial and skin disorder caused by several products. Based on this data, the model suggests a product by analyzing customer's skin using rough set based image processing approach by extracting features that reduce the chances for causing skin or facial disorders that satisfies the customer requirements. As the proposed model supports distributed environment, group of users can use the model for getting best product recommendations. The time levels for analyzing the facial disorders of the customers in the proposed method is compared with the traditional Computer Vision Based Method (CVBM) and the results are depicted in the Figure 3.

Table 1. Customer facial disorder percentage

\begin{tabular}{cccc}
\hline $\begin{array}{c}\text { Age } \\
\text { Group } \\
\text { (in } \\
\text { years) }\end{array}$ & $\begin{array}{c}\text { Annual amount } \\
\text { spent on } \\
\text { Cosmetic } \\
\text { products (in Rs:) }\end{array}$ & Product Type & $\begin{array}{c}\text { Percentage of } \\
\text { cases } \\
\text { identified }\end{array}$ \\
\hline$>15$ & Less than 2,000 & $\begin{array}{c}\text { Skin } \\
\text { moisturizer } \\
\text { product } \\
\text { Facial beauty } \\
\text { product } \\
\text { Facial and } \\
\text { skin beauty } \\
\text { product } \\
\text { Facial beauty } \\
\text { product } \\
\text { Skin rash } \\
\text { avoiding } \\
\text { products }\end{array}$ & $12 \%$ \\
$45-45$ & 2,000 to 4,000 & $18 \%$ \\
\hline & Less than 3,500 & $22 \%$ \\
\hline
\end{tabular}

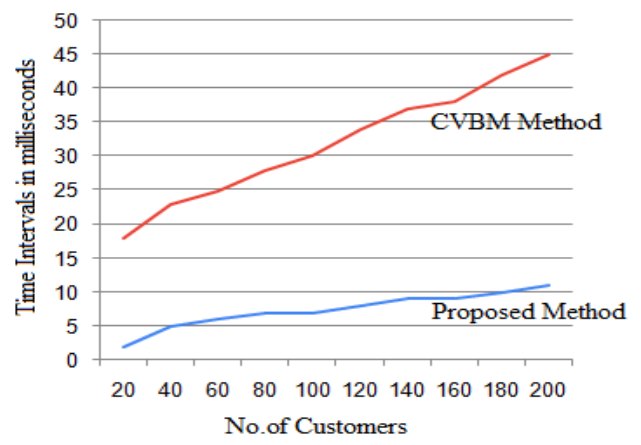

Figure 3. Facial disorder analyzing time intervals

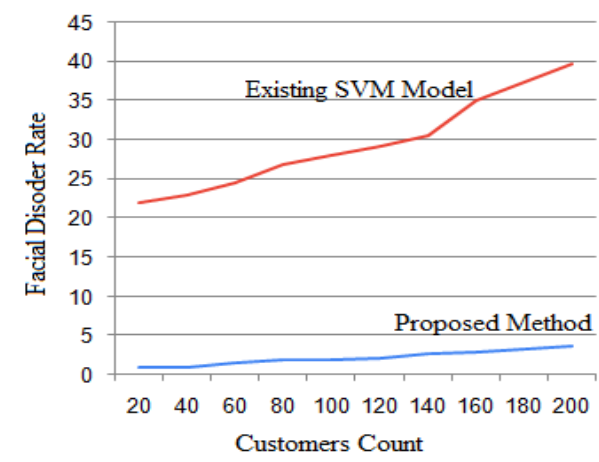

Figure 4. Facial disorder rate after product recommendation

The facial disorder rate of the proposed method is compared with the traditional SVM classifier for product suggestion that causes facial disorders is depicted in Figure 4. The proposed model analyzes the facial features of the customer completely and then identify the exact problem depending on pixel value comparison and similarity. Based on the analysis, the proposed model recommends a suitable product to the customer that reduces the side effects and improves the safety levels of the customer. 
Table 2. Accuracy levels

\begin{tabular}{c|c|c|c|c|c|c}
\hline Model & Product type & $\begin{array}{c}\text { Percentage of } \\
\text { customers }\end{array}$ & $\begin{array}{c}\text { Allergic } \\
\text { Reaction }\end{array}$ & $\begin{array}{c}\text { Chemical } \\
\text { reaction }\end{array}$ & $\begin{array}{c}\text { Distributed } \\
\text { Platform }\end{array}$ & $\begin{array}{c}\text { Accuracy } \\
\text { Accus }\end{array}$ \\
\hline $\begin{array}{c}\text { Existing SVM Model } \\
\begin{array}{c}\text { Proposed rough set based } \\
\text { image processing method }\end{array}\end{array}$ & $\begin{array}{c}\text { Facial Cosmetic } \\
\text { Products } \\
\text { Facial Cosmetic } \\
\text { Products }\end{array}$ & $19 \%$ & Yes & Yes & No & $68 \%$ \\
\hline
\end{tabular}

As the proposed model performs conversion of pixel values into binary format, the comparison and analysis of features will become easy and then suggest a cosmetic product that best fits the skin condition. In this way the facial disorder rate of the proposed model is very low. The accuracy rate in suggesting the cosmetic product to the customers for better results with the proposed method is compared with the traditional methods and the accuracy rate of proposed and traditional methods are depicted in Table 2.

The accuracy levels of the proposed and traditional methods are depicted in the Figure 5. The accuracy of the proposed method is high when compared to traditional methods and the proposed method also available globally as n number of users can request for product recommendation.

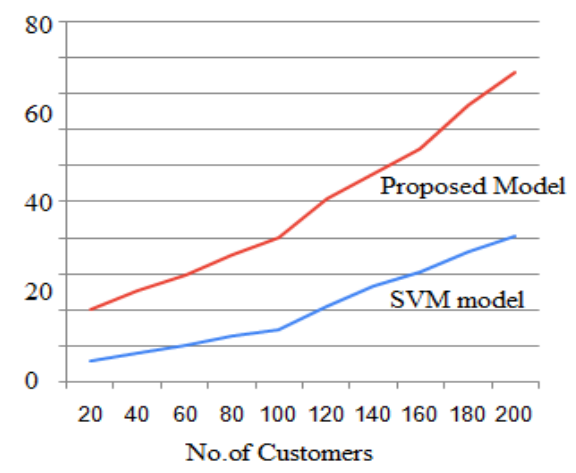

Figure 5. Accuracy level

The proposed model recommends a best cosmetic product that improves the safety levels of the customer. The proposed model analyzes the complete facial features and its dissimilarities and then recommends a product that fits the skin condition. This allows the customer to have a safe condition to avoid side effects. The safety levels of the proposed model are compared with the traditional methods and the results show that the proposed model provides high safety levels. Figure 6 depicts the skin safety levels of the proposed and traditional methods.

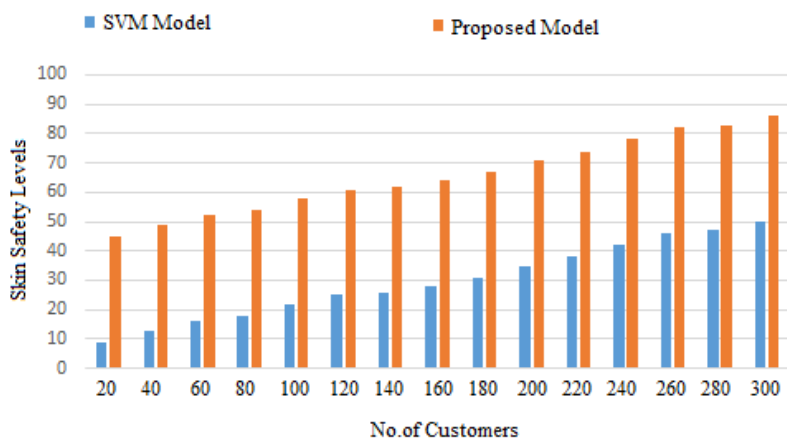

Figure 6. Skin safety levels

\section{CONCLUSION}

Manufacturers of skincare products should lay emphasis on the Research Department for avoiding skin disorders to the customers using those products. Since, the Customers are progressively aware about the symptoms brought about by skincare items. As the utilization of cosmetic products are increasing day by day, manufacture companies have to take much care in producing the cosmetic products that bests suits the customers and satisfy their requirements. The Cosmetic items may introduce disorders and repetitive unfriendly impacts are ascribed to the harmful substances generally found in their definitions. The proposed distributed platform is introduced using the roughest theory and utilizing image processing techniques for recommendation of a product that best suits the skin condition of the customers. The framework is designed as it can be used in any location by predefining and training the model with existing facial disorders caused by several products. The model segments the facial image and analyze the features for recommending a product. The proposed model improves the safety of the customers by reducing the cause of side effects. The proposed model is trained with the local and global features of customers of different regions and then analyze the features and recommends a best product. The model can be used by the customers at local level or in global level for getting better recommendations of the cosmetic product that best suits the skin condition. The proposed method accurately suggests the best cosmetic products to the customers that does not causes any harm to the customers. In future the training time need to be reduced as the proposed model concentrates on local and global features. The number of dataset samples can also be increased to improve accuracy levels also.

\section{REFERENCES}

[1] Patty, J.C., Kirana, E.T., Giri, M.S.D.K. (2017). Recommendations system for purchase of cosmetics using content-based filtering. International Journal of Computer Engineering and Information Technology, 10(1): $1-5$.

[2] Ekstrand, M.D., Riedl, J.T., Konstan, J.A. (2011). Collaborative Filtering Recommender Systems. Foundations and Trends $®$ in Human-Computer Interaction, 4(2): 81-173.

[3] Sharma, L., Gera, A. (2013). A survey of recommendation system: Research challenges. International Journal of Engineering Trends and Technology (IJETT), 4(5): 1989-1992.

[4] Yasir, R., Nibir, M.S.I., Ahmed, N. (2015). A skin disease detection system for financially unstable people in developing countries. Global Science and Technology Journal, 3(1): 77-93.

[5] Ghazanfar, M., Prügel-Bennett, A. (2010). Building 
switching hybrid recommender system using machine learning classifiers and collaborative filtering. IAENG International Journal of Computer Science, 37(3).

[6] Bhatia, L., Prasad, S.S. (2015). Building a distributed generic recommender using scalable data mining library. 2015 IEEE International Conference on Computational Intelligence \& Communication Technology, Ghaziabad, pp. 98-102. https://doi.org/10.1109/CICT.2015.129

[7] Draelos, Z.D. (2015). Cosmetics: The medicine of beauty. Journal of Cosmetic Dermatology. 14(2): 91. https://doi.org/10.1111/jocd.12146

[8] Schneider, G., Gohla, S., Schreiber, J., Kaden, W., Schomock, U., Lewerkuhne, H.S., Kuschel, A., Petsitis, X., Pape, W., Ippen, H., Diembeck, W. (2001). Skin cosmetics. Ullmann's Encyclopedia of Industrial Chemistry, Germany: Wiley $\mathrm{VCH}$, 6: 24-29. https://doi.org/10.1002/14356007.a24_219

[9] Portugal, I., Alencar, A., Cowan, D. (2018). The use of machine learning algorithms in recommender systems: A systematic review. Expert Systems with Applications, 97: 205-227.

[10] Akay, B., Kaynar, O., Demirkoparan, F. (2017). Deep learning based recommender systems. 2017 International Conference on Computer Science and Engineering (UBMK), Antalya, pp. 645-648. https://doi.org/10.1109/UBMK.2017.8093489

[11] Robertson, J., Fieldman, G., Hussey. T.B. (2008). Who wears cosmetics?" Individual differences and their relationship with cosmetic usage. Individual Differences
Research, 6(1): 38-56.

[12] Rinaldi, A. (2008). Healing beauty? More biotechnology cosmetic products that claim drug-like properties reach the market. EMBO Reports, 9(11): 1073-1077. https://doi.org/10.1038/embor.2008.200

[13] Dooms-Goosens, A. (1993). Cosmetics as causes of allergic contact dermatitis. Cutis, 52(5): 316-320

[14] Donsing, P., Viyoch, J. (2008). Thai Breadfruit's heartwood extract: A new approach to skin whitening. Srinakharinwirot Science Journal, 24 (1): 9-23.

[15] Sattar, A., Ghazanfar, M.A., Iqbal, M. (2017). Building accurate and practical recommender system algorithms using machine learning classifier and collaborative filtering. Arabian Journal for Science and Engineering, 42(8): 3229-3247.

[16] Nigam, P.K., Saxena, A.K. (1988). Allergic contact dermatitis from henna. Contact Dermatitis, 18(1) :55-56.

[17] Durmazlar, S.P., Tatlican, S., Eskioglu, F. (2009). Localized hypertrichosis due to temporary henna tattoos: Report of three cases. Journal of Dermatological Treatment, 20(6): 371-373. https://doi.org/10.3109/09546630802691945

[18] D’Arcy, P.F. (1982). Fatalities with the use of henna dye. Pharmacy International, (3): 217-218

[19] Johansen, J.D., Andersen, K.E., Rastogi, S.C., Menne, T. (1996). Threshold responses in cinnamic-aldehydesensitive subjects: Results and methodological aspects. Contact Dermatitis, 34(3): 165-171. https://doi.org/10.1111/j.1600-0536.1996.tb02167.x 